Revue des patrimoines

\title{
La conservation des voitures hippomobiles des musées de province. Le cas des voitures hippomobiles, objet isolé au sein d'une collection
}

Nathalie Giffard de la Jaille

\section{(2) OpenEdition}

Journals

Édition électronique

URL : http://journals.openedition.org/insitu/9650

DOI : 10.4000/insitu.9650

ISSN : 1630-7305

Éditeur

Ministère de la culture

Référence électronique

Nathalie Giffard de la Jaille, "La conservation des voitures hippomobiles des musées de province. Le cas des voitures hippomobiles, objet isolé au sein d'une collection », In Situ [En ligne], 18 | 2012, mis en ligne le 31 juillet 2012, consulté le 02 mai 2019. URL : http://journals.openedition.org/insitu/9650 ; DOI : 10.4000/insitu.9650

Ce document a été généré automatiquement le 2 mai 2019.

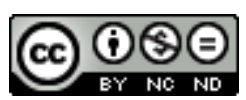

In Situ Revues des patrimoines est mis à disposition selon les termes de la licence Creative Commons Attribution - Pas d'Utilisation Commerciale - Pas de Modification 4.0 International. 


\title{
La conservation des voitures
} hippomobiles des musées de province. Le cas des voitures hippomobiles, objet isolé au sein d'une collection

\author{
Nathalie Giffard de la Jaille
}

\section{Introduction}

1 Regard et analyse d'une passionnée d'attelage, meneur de tradition, juge FFE à travers son expertise de restaurateur du patrimoine, section peinture, exerçant depuis plus de vingt ans dans les Musées de France ${ }^{1}$ (Agrément DMF, membre : FFCR, SFIIC).

2 Collectionneuse de voitures anciennes, c'est donc tout naturellement que ce patrimoine a attiré mon attention lors de mes divers déplacements dans les musées de province, châteaux-musées, écomusées. Enfin ce double statut de technicien de la restauration/ meneur-collectionneur permet de poser un regard croisé sur les pratiques actuelles en matière de restauration de ce type de patrimoine.

3 Cet état des lieux n'a d'autre prétention que d'attirer l'attention sur une situation préoccupante pour ne pas dire alarmante dans certains cas.

\section{Le problème du stockage}

4 Les voitures qui nous intéressent ici sont effectivement stockées dans des conditions assez rudimentaires pour la plupart d'entre elles. On ne parle plus donc de remisage mais malheureusement pas non plus de mise en réserve.

5 Autrefois « remisées ", on parle en effet de remise à voitures quand il s'agit de voitures hippomobiles ainsi que de sellerie en ce qui concerne les harnais. 

a relégation " dans des locaux non appropriés, presque toujours multifonctions. Bon nombre d'entre elles sont en effet stockées dans des bâtiments, type ateliers municipaux, garages pour véhicules automobiles en fonction, locaux industriels désaffectés etc. Ce sont des locaux, clos et hors d'eau, fermés à clef mais non sécurisés. Les risques de vols ou de dégradation par actes de vandalisme ne sont pas les majeurs, il n'en est pas de même des risques de cassures, écrasements accidentels liés aux diverses manœuvres et manipulations de véhicules ou d'objets également stockés dans ces lieux. Les conditions climatiques étant naturelles, sans contrôle, participent hélas au vieillissement des matériaux.

Enfin un incendie serait le plus souvent fatal, tant sortir certaines voitures est impossible, non pas à cause de leur poids et inertie mais à cause de leur inaccessibilité et de l'encombrement des locaux.

On le voit donc, les voitures sont loin de bénéficier des mesures conservatoires dont peut jouir le reste des collections. On entend par là une mise en réserve dans des locaux adéquats : sécurisés, contrôlés d'un point de vue climatique.

\section{Quelques cas représentatifs}

\section{Le Coupé-dormeuse du Château-Musée de Saumur, fin $\mathrm{XIX}^{\mathrm{e}}$ siècle}

13 Imposante berline de voyage, voiture à quatre roues, donnée à l'association des Amis du Musée du Château de Saumur au début du XXe siècle, appartint d'après les archives du Musée, à la famille de Brézé en Anjou (fig. nº1). 
Figure 1

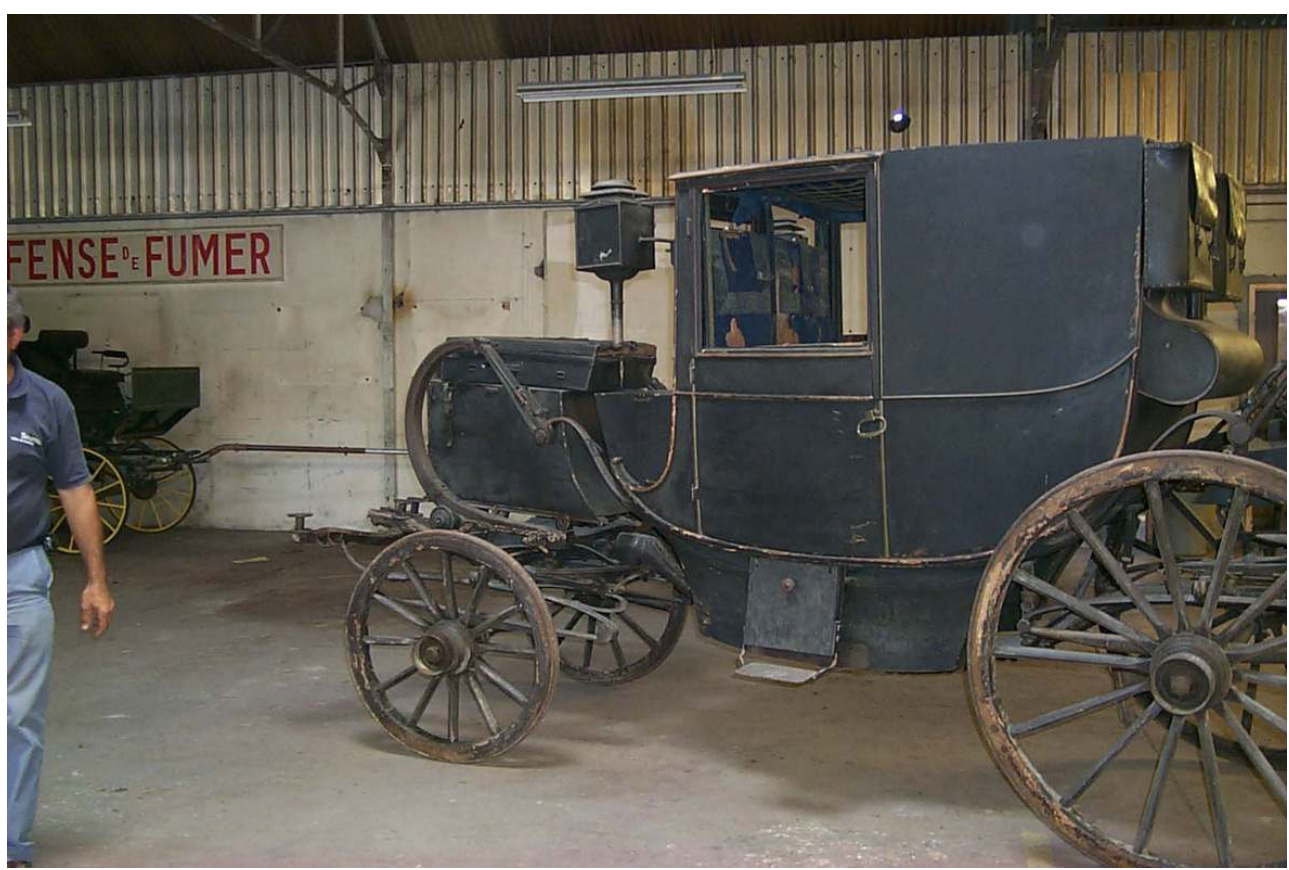

Le coupé-dormeuse du Château-Musée de Saumur à son arrivée à Saumur.

(c) Château-Musée de Saumur.

Faute de place au Château de Saumur, elle fut mise en dépôt au Musée des Transports de Blois dans les années soixante-dix et à la fermeture définitive de celui-ci, renvoyée à Saumur en 2007.

Toujours en l'absence de lieu de présentation à Saumur, la voiture est stockée dans un local industriel désaffecté à l'usage des services techniques de la ville. Elle repose sur un sol en ciment, bâchée contre la poussière et les risques de fuite d'eau accidentels. Les premières constatations à son arrivée confirment un état général assez exceptionnel, un état de présentation encore très satisfaisant pour un état d'origine ou proche ; on note la présence en parfait état des grandes lanternes signées d'un manufacturier parisien renommé.

16 Ces constatations font aussi malheureusement apparaître l'absence du timon, pièce maîtresse d'une voiture qui bien qu'amovible, ne fait qu'un avec elle. Les recherches effectuées immédiatement par la conservation du Château de Saumur auprès de la ville de Blois, responsable du Musée des Transports, s'avèrent hélas infructueuses (fig. $\mathbf{n}^{\circ} \mathbf{2}$ ). 
Figure 2

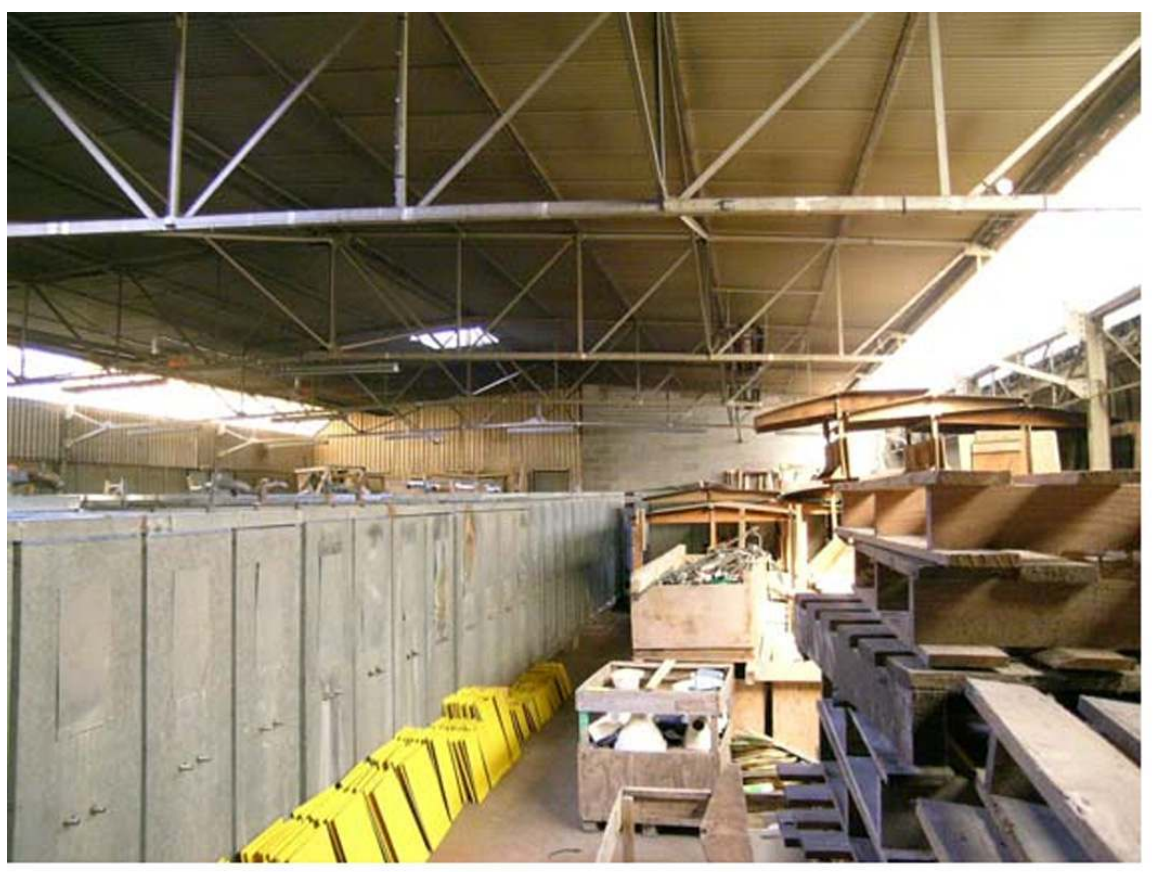

Le coupé-dormeuse du Château-Musée de Saumur. Vue du site de stockage.

(c) Château-Musée de Saumur.

17 Le timon sera découvert tout à fait fortuitement quelques années plus tard, lors d'une discussion avec un sellier lors d'une manifestation d'attelage de tradition. Il était demeuré dans l'atelier lors d'une intervention sur la voiture.

La deuxième mauvaise surprise intéresse un des éléments de la voiture, on note en effet le changement (reconstruction à neuf) de la capote arrière. La conservation du Château de Saumur, propriétaire de l'objet, ne peut que le constater n'ayant jamais été informée d'une intervention sur son objet. Celle-ci a été effectuée par la sellerie qui avait conservé le timon, mais malheureusement il n'existe pas de dossier de restauration.

Ce cas particulier est intéressant parce qu'il est très représentatif du statut de ce type d'objets au sein des musées, les décennies passées : il met en lumière la façon dont ils ont

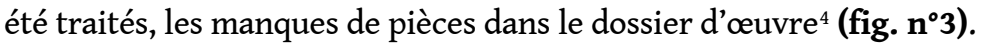


Figure 3

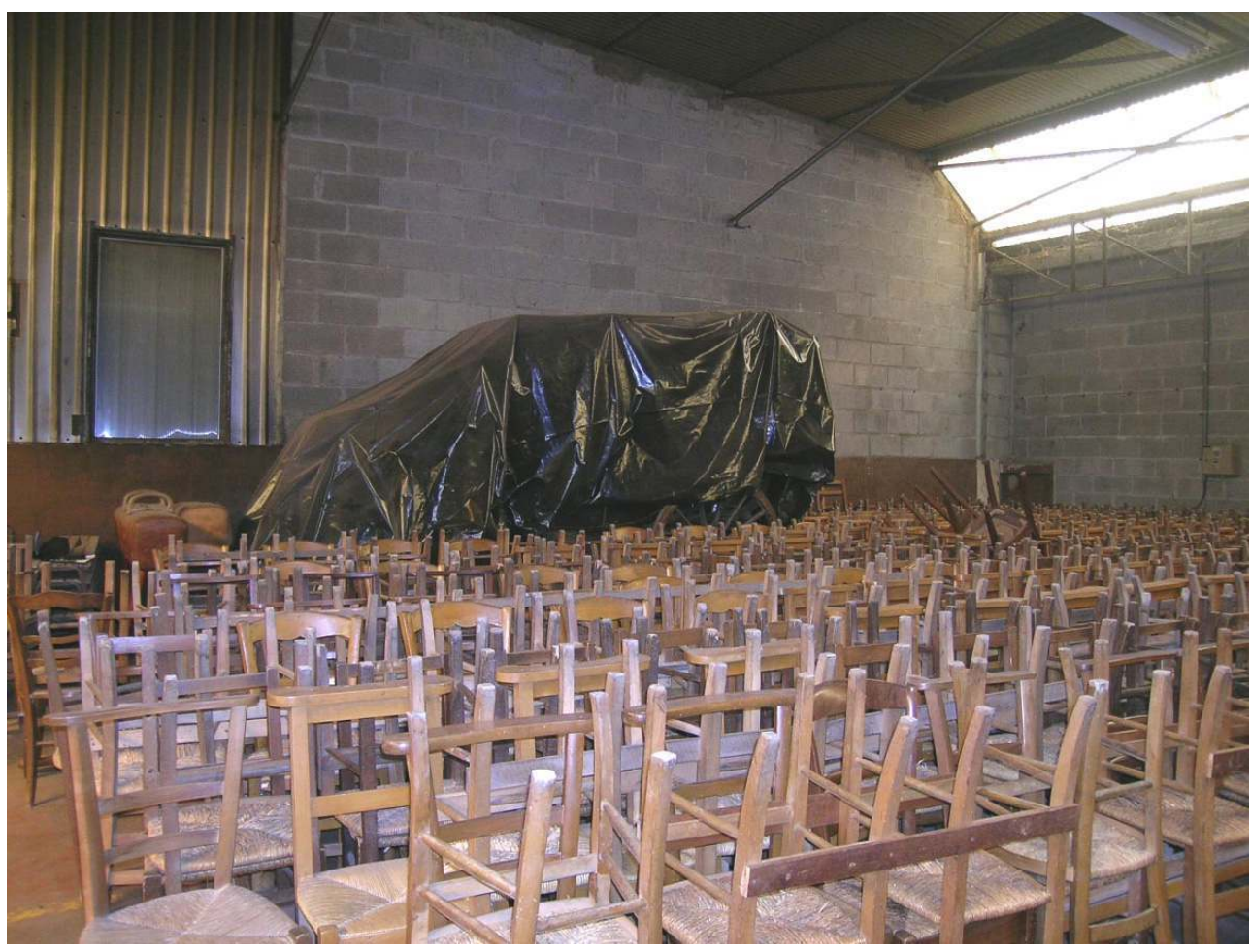

Le coupé-dormeuse du Château-Musée de Saumur. La voiture sous bâche.

(c) Château-Musée de Saumur.

20 Ce patrimoine hippomobile est, comme le patrimoine aéronautique, maritime, lié à une pratique particulière, victime d'une méconnaissance du domaine mais peut-être aussi hélas d'un désintérêt voire d'un manque de considération pour la chose technique.

L'intervention sur la capote souligne également l'absence de concertation et d'autorisation entre le musée dépositaire et le musée propriétaire de l'objet, manquement préjudiciable car cette concertation aurait pu permettre de valider, lors d'une commission de restauration, le bien-fondé de cette intervention.

\section{La berline-coupé du Musée Henri Barré de Thouars, dernier quart du $\mathrm{XVIII}$ siècle}

22 Entrée dans les collections du musée, cette voiture à quatre roues, berline-coupé de Monsieur de Lignières, date du XVIII ${ }^{\mathrm{e}}$ siècle. Elle est, tant par sa typologie que par sa mise en œuvre (caisse peinte et dorée, train bois doré) particulièrement remarquable, c'est un exemplaire rare $^{5}$. Elle est dans son état d'origine ou proche, son aspect de présentation est moyen à présentable, la lisibilité de son décor peint est encore très satisfaisante. On déplore la perte du siège de cocher et l'absence du timon (fig. $\left.\mathbf{n}^{\circ} \mathbf{4}\right)$. 
Figure 4

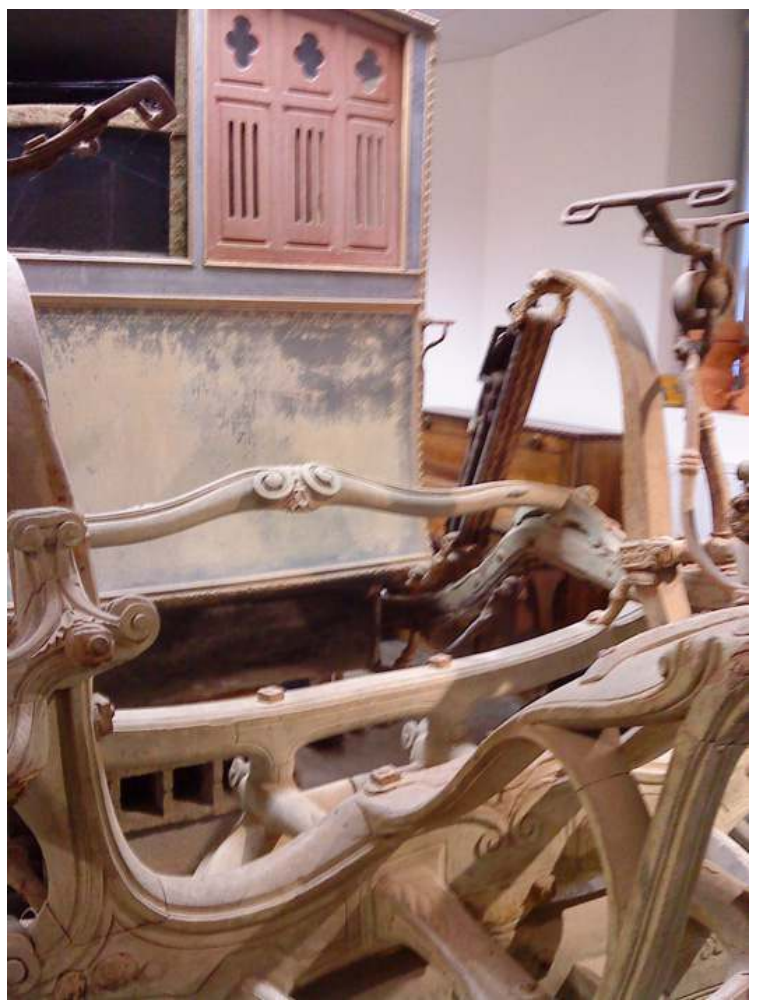

Le coupé-berline du Musée de Thouars, détail.

Phot. N. Giffard de la Jaille. (c) N. Giffard de la Jaille.

Cette voiture, faute de place, n'est pas exposée au public, elle est stockée dans un petit local et difficilement accessible. Ce local jouxte le musée, il est parfaitement sécurisé contre le vol, les dégradations ou les dégâts des eaux mais quid de son extraction en cas d'incendie, tant le lieu est encombré d'objets ? (fig. $\mathbf{n}^{\circ} 5$ ) 


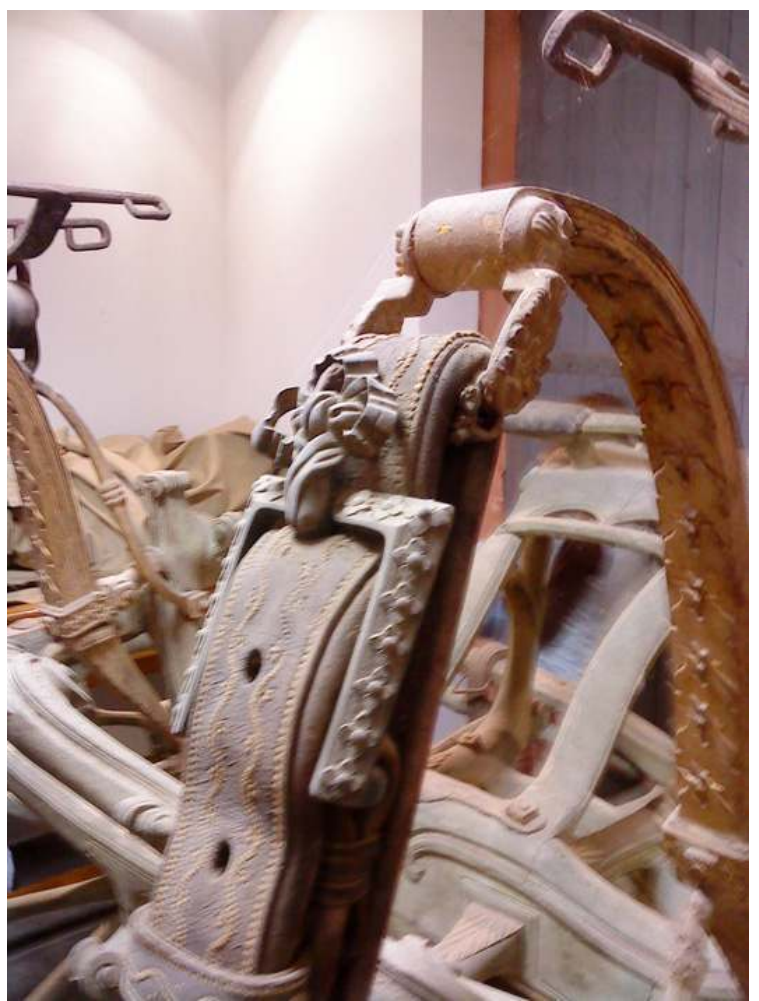

Le coupé-berline du Musée de Thouars, détail.

Phot. N. Giffard de la Jaille. (c) N. Giffard de la Jaille.

En ce qui concerne cette voiture, le risque le plus préoccupant, compte tenu de sa mise en œuvre (bois doré sculpté) est davantage le déséquilibre climatique du lieu. La " remise » est installée dans une annexe du musée recevant des scolaires en atelier pédagogique, donc un ensemble bien chauffé. Une atmosphère chaude et sèche est préjudiciable pour ce type de matériaux, entraînant la déshydratation des bois et, ici, des pertes de matière sur la dorure. La voiture n'est pas bâchée par un voile contre la poussière; sa caisse, présentant un affaiblissement de structure, est étayée sommairement.

\section{Le fourgon du Musée du textile de Cholet, fin XIXe siècle}

Entré dans les collections du Musée du textile, ce fourgon de livreur d'écheveaux de fils aux filatures de Cholet est intéressant non seulement par sa typologie (peu de voitures utilitaires sont encore conservées) mais surtout parce que son histoire et sa provenance sont connues. Cette voiture, état d'origine ou proche, possède en effet encore ses plaques d'identification : nom, adresse, métier du propriétaire (fig. $\mathbf{n}^{\circ} \mathbf{6}$ ). 
Figure 6

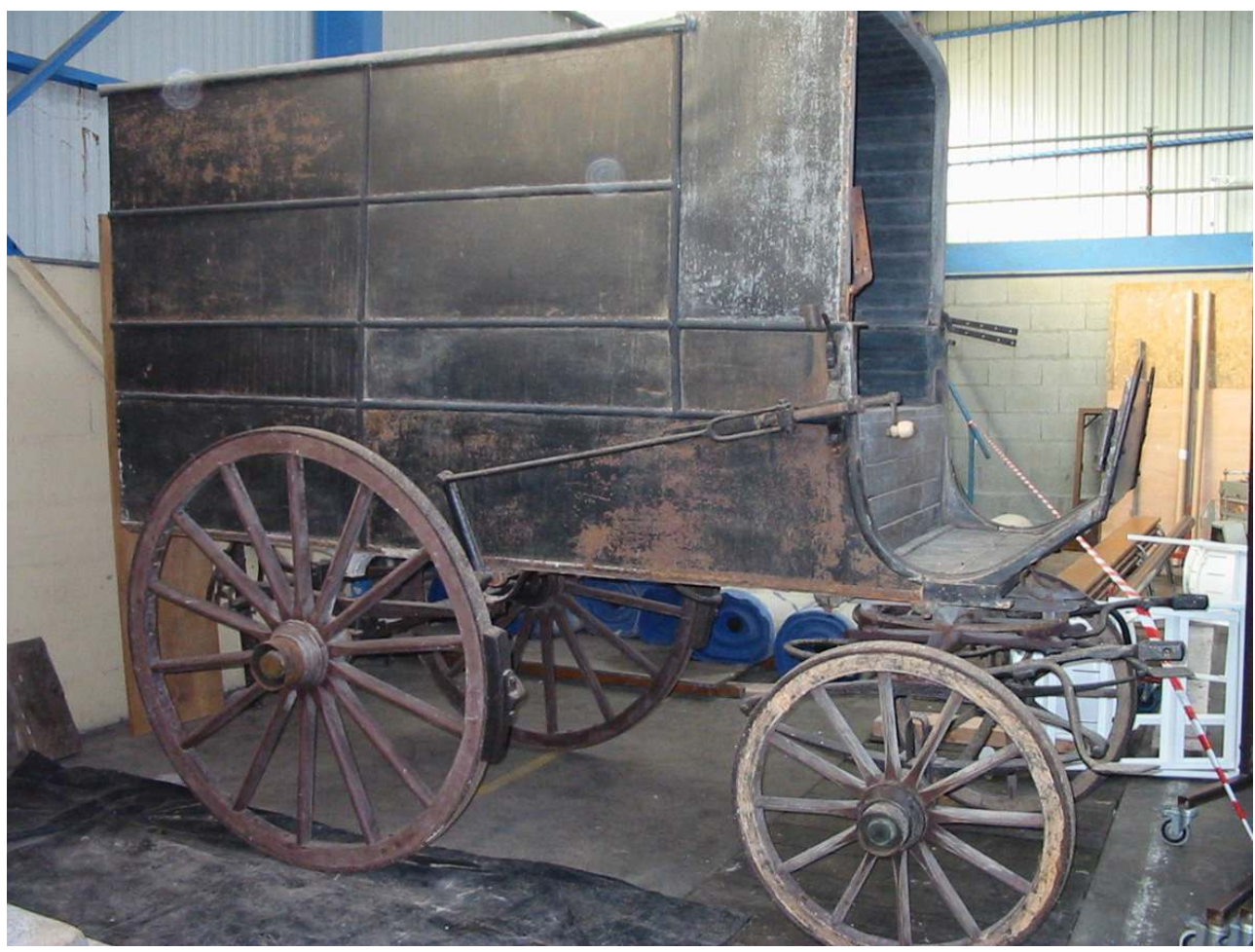

Le fourgon du Musée du textile de Cholet. Vue dans le local.

Phot. N. Giffard de la Jaille. (c) N. Giffard de la Jaille.

Voiture fermée à quatre roues, elle est complète, stockée avec sa limonière ${ }^{6}$ désolidarisée. Son aspect est moyen à passable, elle est encore présentable en l'état (fig. $\mathbf{n}^{\circ} 7$ ). 
Figure 7

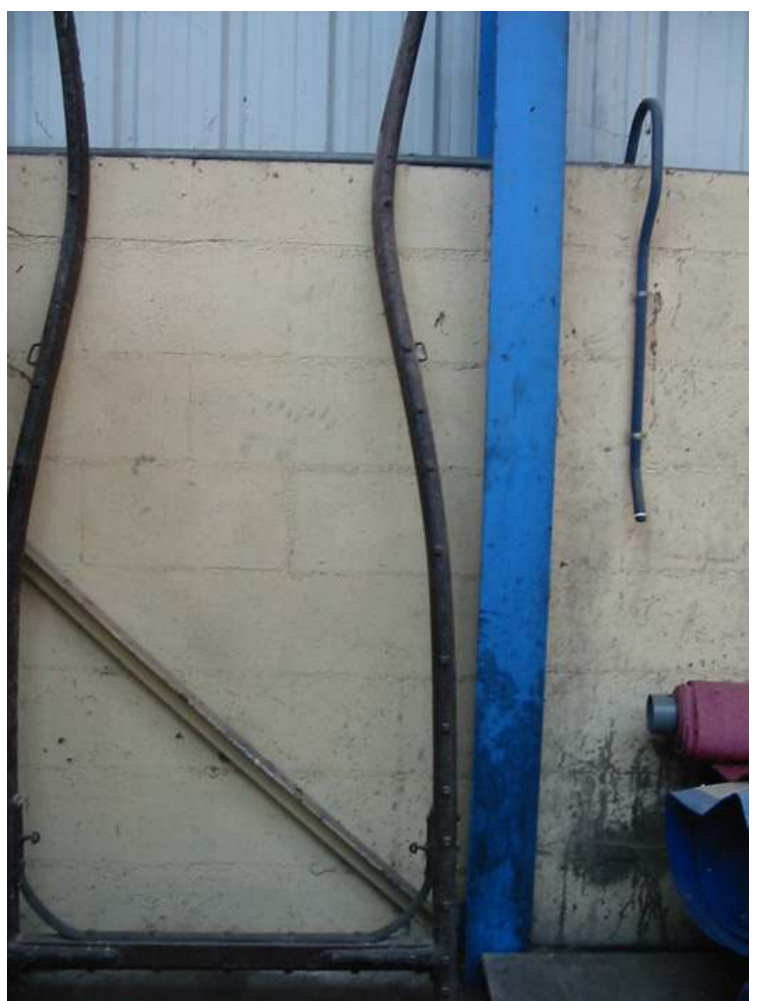

Le fourgon du Musée du textile de Cholet. La limonière.

Phot. N. Giffard de la Jaille. (c) N. Giffard de la Jaille.

Malheureusement, là encore faute de place au sein du musée et bien qu'en lien direct avec la collection qui présente des métiers à tisser en action, cette voiture n'est pas exposée au public. Il a été envisagé un temps une exposition temporaire à la belle saison sous auvent dans l'enceinte du musée, mais diverses contraintes ont fait que cette opération n'a jamais vu le jour. Elle est stockée dans un local municipal, bâtiment de stockage industriel désaffecté, un peu à l'écart de la ville, donc sans inspection régulière des lieux; elle partage avec une autre voiture utilitaire ce local avec des objets lourds et encombrants entrainant une situation à risque lors des manipulations de ceux-ci. Les conditions de ce local sont naturelles, le sol est en ciment, la voiture n'est pas bâchée contre la poussière (fig. $\left.\mathbf{n}^{\circ} \mathbf{8}\right)$. 


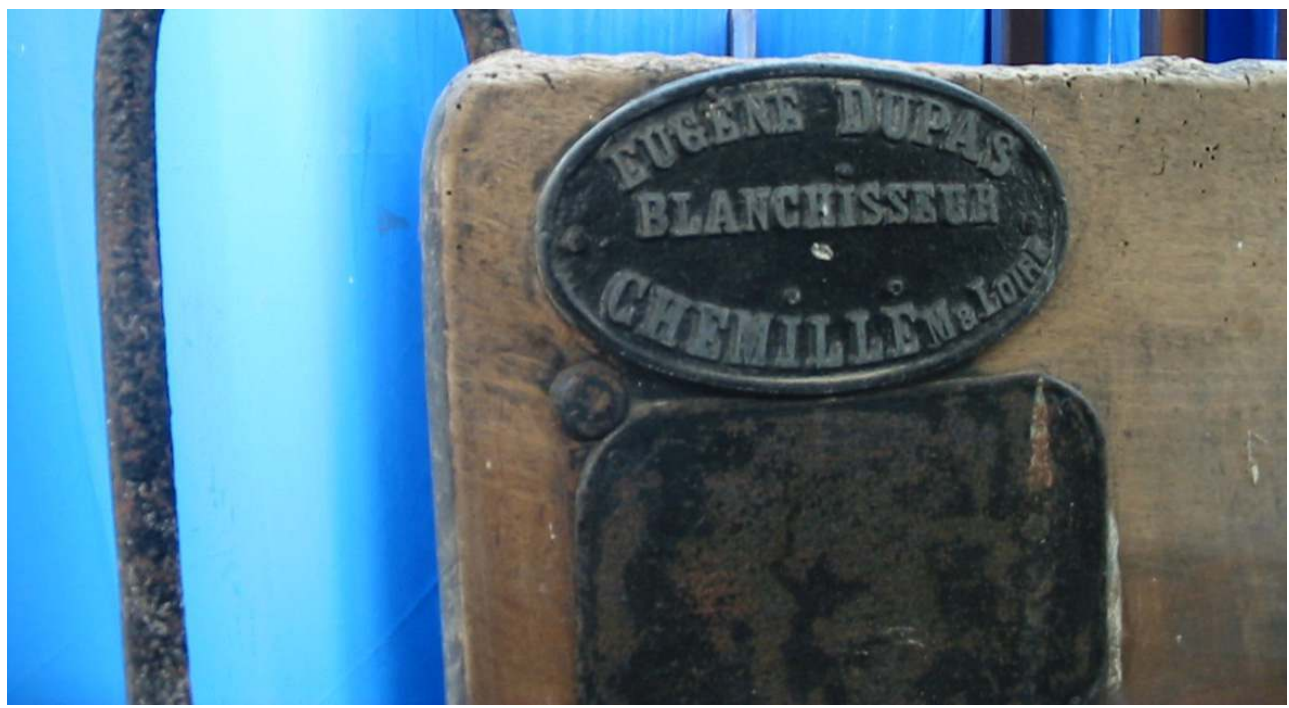

Le fourgon du Musée du textile de Cholet. Les plaques d'identification.

Phot. N. Giffard de la Jaille. (c) N. Giffard de la Jaille.

\section{Le Coupé ou « demi-fortune » du Musée des Transports de Toulouse}

Informations communiquées par le conseiller musées de la Direction régionale des affaires culturelles (DRAC) Midi-Pyrénées dans le cadre du colloque de Tulle.

La collection du musée a été formée au départ par une association puis est entrée sous la tutelle des Musées de France ; elle présente plusieurs types de véhicules.

En ce qui concerne les voitures hippomobiles, outre le coupé7, voiture à quatre roues, fin $\mathrm{XIX}^{\mathrm{e}}$ siècle, qui retient ici notre attention, on note une voiture de parc en osier, un cabriolet, une chaise à porteurs et quatre voitures agricoles communes ; ces voitures ont toutes fait l'objet d'un constat d'état du C2RMF en 2000 avec un rapport d'Odile Lecomte qui précisait déjà que «les véhicules sont éparpillés dans trois lieux inappropriés, qui sont à l'origine des plus gros dégâts que l'on trouve sur les voitures ».

Là encore, en l'absence d'espace de présentation, de réserves adéquates, les voitures sont restées dans des garages municipaux, et lors d'une manœuvre d'un véhicule automobile, le coupé a été accidenté et les débris évacués.

31 Ce cas particulier montre que, malgré la bonne volonté de tous, cet objet inventorié, étudié par le service de restauration des Musées de France a été détruit.

\section{Les contraintes et conditions de conservation liées à ce type d'objet}

Il est aisé de constater que « dévoreur d'espace ", ce type d'objet n'est guère présenté au public excepté dans certaines structures en lien avec la pratique de l'attelage comme les Haras nationaux ou certains monuments nationaux comme les châteaux de Versailles, Chambord ou Compiègne. 

climatiques (chaleur importante sous des tôles ondulées par exemple), il peut être envisagé un «boitage de protection », c'est une ossature en bois légère pouvant supporter différents matériaux isolants et imperméables. Ce boîtage doit être envisagé sans contact avec la voiture, accessible pour une inspection et le plus contrôlable possible pour y gérer une stabilité climatique, souvent par apport d'humidité l'été par temps sec. dépoussiérage, du soutien des parties portantes, bas de caisse, etc., voire de petites mesures de consolidation ou renfort d'urgence de parties trop affaiblies. Bien qu'il ne s'agisse pas de restauration à proprement parler, certaines interventions doivent faire l'objet de concertations pluridisciplinaires; les voitures nous l'avons vu étant plurimatériaux. 
42 Dans tous les cas de figure, ces mesures simples de conservation préventive doivent s'accompagner d'une inspection régulière du site et de l'objet: elle devra être effectuée plusieurs fois par an, si possible par le même personnel, sensibilisé à ce type de patrimoine. Cette inspection devra particulièrement veiller à l'aspect climatique du site : infiltration, ruissellement d'eau sous les portes en cas d'orage mais aussi chaleur sèche excessive (le risque majeur pour une voiture étant la déshydratation des bois).

Ces mesures de conservation préventive peuvent être également adoptées et adaptées aux périodes d'hivernage des collections de voitures des sites fermés l'hiver (collections de châteaux publics ou privés).

\section{Conservation-restauration, conservation préventive. Reconstruction à l'identique, copie d'ancien...}

Comme le souligne très pertinemment, le conseiller musées de la DRAC Midi-Pyrénées, en matière de conservation et restauration, ce patrimoine hippomobile est assez similaire à d'autres patrimoines : patrimoine aéronautique, maritime etc. Patrimoines en lien avec une technologie complexe et des pratiques encore vivantes, ils posent tous les mêmes questionnements, les mêmes problèmes.

Enfin ce patrimoine est bien évidemment traité selon son statut: public, associatif ou privé. Les Musées de France et les collectionneurs privés ont des approches, des motivations, des pratiques et des courants de pensée différents.

Concernant les musées, la politique de la direction des Musées de France est simple et cohérente : protéger et sauvegarder au maximum les matériaux d'origine, par là-même les savoir-faire anciens. Les interventions actuelles quel que soit le type de patrimoine, se veulent en accord avec la déontologie de la conservation-restauration en place tant en France qu'en Europe depuis quelques décennies, à savoir tendre à et respecter les trois grandes règles de la restauration moderne : stabilité, réversibilité et lisibilité.

Le positionnement est donc clair quand il s'agit de refaire certaines parties manquantes, on préfère alors "rapporter » à l'original des matériaux hétérogènes (de natures différentes) facilement identifiables des matériaux d'origine. Ce choix est primordial en effet, que pourront étudier les générations futures de chercheurs, historiens, si on ne leur transmet plus, ou de moins en moins de matériaux anciens et de mise en œuvre anciennes?

8 Autre point fondamental, ces voitures rattachées à des collections publiques sont des objets de musée, elles ne sont donc plus considérées comme roulantes, c'est-à-dire qu'elles ne doivent plus être mises en situation d'être attelées (et ce quelle que soit la pression que pourrait exercer un tiers). Cette orientation est, là encore, primordiale, elles n'ont plus ainsi à être restaurées pour être sécurisantes. Ce qui ne veut pas dire que la structure portante ne doit pas être conservée en état de résistance mécanique, l'objet doit pouvoir être déplacé sans risques de " désossement " $^{8}$.

La conservation préventive, quant à elle, est justifiée pour les voitures citées ci-dessus, leur « devenir » n'étant pas décidé. Les restaurer pour les rendre présentables n'est pas une priorité, si aucun lieu n'est disponible. En matière de conservation-restauration, comme dans d'autres domaines, nous faisons sans cesse des avancées, des progrès... il n'est donc pas déraisonnable de se donner un temps de réflexion. 
50 Le courant "Attelage de Tradition» est lui fondé sur la sauvegarde du patrimoine hippomobile ancien à travers la pratique du «menage » et de ses traditions. Le menage est un terme consacré pour parler de l'art de mener des chevaux et on entend par traditions de cette discipline la dextérité du meneur, l'endurance de l'équipage et l'élégance de l'ensemble.

51 «Pratique vivante", ici le mot «vivante», sans jeu de mots, prend un réel sens! L'attelage est une discipline dangereuse, sa pratique doit donc se faire dans le plus grand respect des règles de sécurité. La sécurité passant dans une voiture hippomobile par une caisse et un train sains et solides, il va sans dire qu'il en est de même pour le harnais.

Se pose alors, compte tenu de tous ces impératifs, le degré d'intervention lors d'une restauration et le choix n'est ni simple, ni aisé à mettre en œuvre. Une voiture est une construction complexe pleine d'interactions, "toucher » une partie, opérer sur un type de matériau entraînant presque toujours une intervention sur un autre matériau. À titre d'exemple, la consolidation d'une caisse ${ }^{9}$ passe presque inévitablement par un problème de peinture ou de vernis.

La « Note Présentation » est également très importante lors des concours de tradition, la propreté du matériel, son état et l'élégance de l'équipage étant un des paramètres de notation, la majorité des adeptes de ces concours s'investissent beaucoup pour rivaliser d'harmonie.

54 Ces grandes orientations posent une foule de questionnements... mais une seule réponse existe : transmettre ce patrimoine et comme chaque cas est particulier, la concertation devrait être le maître mot!

Cruel dilemme, en effet est-ce qu'une voiture entièrement démontée et reconstruite est encore un objet ancien? Une voiture est considérée comme ancienne jusqu'à quel pourcentage d'interventions? Nos interventions actuelles si bien réalisées soient-elles, tant d'un point de vue technique qu'esthétique, peuvent-elles être validées comme des savoir-faire anciens ?... Ne nous leurrons pas, malgré tout le respect et le soin que l'on peut apporter à ces restaurations, nous n'avons plus les mêmes pratiques (nous mécanisons la plupart de nos interventions par exemple) et hélas nous ne trouvons plus sur le marché les mêmes qualités de matières premières.

56 Le sujet, on le devine, on le sait, n'est pas simple, il divise même! Cependant des pistes de réflexion voient le jour, l'Association Française d'Attelage, AFA, à qui l'on doit le renouveau de cette pratique et le sauvetage de voitures anciennes, tente de structurer le domaine.

\section{Au sujet de la piste des copies}

57 Atteler une « copie d'ancien » est une piste mais ne résout que partiellement le problème. Elle est la solution pour une pratique sportive « musclée », comme les tests de maniabilité ${ }^{10}$ en épreuves fédérales de la FFE. Les voitures de présentation peuvent ainsi offrir le maximum de sécurité (elles sont pourvues de freins à disques, etc.) en accord avec un aspect esthétique irréprochable. Nul besoin donc de trahir une voiture ancienne par l'adjonction d'une technologie qu'elle n'a jamais arborée.

Mais une reconstruction, aussi belle soit-elle, reste une copie et qu'en est-il du patrimoine ancien s'il n'est plus exploité ? N'est-il pas à craindre qu'il soit alors à nouveau délaissé ? Consciente de toutes ces difficultés, l'AFA autorise les copies dans ses épreuves (seule la 
notation diffère), les tenants de la tradition crient au scandale... cependant n'est-ce pas une piste si elle peut sauver d'une restauration invasive certains types de véhicules rares?

\section{Conclusion}

Si les grandes collections publiques prestigieuses, centralisées, sont à l'abri, la situation dans les musées de province, on le voit, reste préoccupante. Le statut d'objet de musée ne suffit pas toujours à protéger ce type de patrimoine (exemple le coupé du Musée des Transports de Toulouse, détruit accidentellement et évacué).

Il est donc urgent de mettre en place une politique générale de sauvegarde et, comme le préconise le conseiller musées de la DRAC Midi-Pyrénées, provoquer une rencontre entre responsables du service des collections et l'inspection générale des patrimoines afin de les informer de la situation globale des voitures hippomobiles et « autres engins » dans les collections.

Ce regard de passionnée ne s'arrête pas aux seuls objets de musée, la sonnette d'alarme pourrait être tirée pour les collections des châteaux-musées, les collections d'écomusées tant publiques que privées.

Les Haras nationaux quant à eux, ont toujours possédé leur propre fonctionnement, lié à l'exploitation attelée des voitures mais cette situation restera-t-elle pérenne face à une baisse de pratique?

\section{NOTES}

1. - Un grand merci aux responsables des collections : conseillers musées, conservateurs et régisseurs bien conscients de leur valeur patrimoniale, sans lesquels je n'aurais pu réaliser ce petit état des lieux. Tous acteurs soucieux de sa conservation, malheureusement toujours aux prises avec une matérialité prégnante.

2. - Timon : longue pièce de bois fixée dans l'axe de l'avant train, participant à la traction et servant à relier les chevaux à la voiture.

3. - GUILLEMARD, Denis \& LAROQUE, Claude. Conservation préventive, gestion et contrôle de collections. $2^{\circ}$ édition. OCIM/DRAC Bourgogne, 1991.

4. - Dossier d'œuvre : dossier propre à chaque œuvre d'une collection, compilation des études, données historiques, interventions de restauration, en deux mots « la traçabilité de l'objet ».

5. - Voir l'article de Jean-Louis Libourel la concernant dans : Voitures hippomobiles, Vocabulaire, typologique et technique. Paris: Monum, éditions du patrimoine, 2005 (coll. Principes d'analyse scientifique).

6. - Brancards solidarisés par une traverse fixe, permettant d'atteler un cheval à une voiture à quatre roues ; ici structure en métal, légèrement cintrée pour atteler un deuxième cheval lorsque la voiture était lourdement chargée.

7. - Coupé ou demi-fortune : voiture pour le transport des personnes, à l'usage d'un particulier, à un cheval de sang; elle est partiellement démontable et transformable en voiture couverte ou 
découverte selon la saison; il en existe deux exemplaires au Musée de Compiègne (information donnée par Jean-Louis Libourel).

8. - CORÉ, Conservation et restauration du patrimoine culturel. Co-édition SFIIC/ERRANCE $n^{\circ} 11$, décembre 2001.

9. - Caisse : corps de la voiture, fabriquée en bois. Elle est la partie destinée à recevoir les passagers ou les marchandises transportés.

10. - Test de vitesse et de dextérité, entraînant le passage d'une combinaison de portes au chrono.

INDEX

Mots-clés : restauration, conservation préventive, attelage, attelage de tradition, calèche, carrosse

\section{AUTEUR}

\section{NATHALIE GIFFARD DE LA JAILLE}

restauratrice, Atelier CRA, Angers, delajaille@orange.fr 